\section{Nichtkoitale HPV-Übertragung bei adoleszenten Mädchen}

Neben dem vaginalen Geschlechtsverkehr muss auch nichtkoitaler Sexualkontakt als wichtiger Übertragungsweg für humane Papillomviren (HPV) angesehen werden. In einer Studie waren 45\% der adoleszenten Mädchen bereits vor dem ersten vaginalen Geschlechtsverkehr mit HPV-Typen infiziert. Diese neuen Befunde legen eine Vorverlegung des HPV-Impfzeitpunkts nahe.

$B^{\mathrm{i}}$ islang galt vaginaler Geschlechtsverkehr als Hauptübertragungsweg für eine Infektion mit dem humanen Papillomvirus (HPV). Allerdings lässt sich HPV manchmal auch in der genitalen und oralen Mukosa bei Frauen nachweisen, die keinen Geschlechtsverkehr hatten; die Übertragungswege sind ungeklärt. Die Aussagekraft der meisten Studien ist zudem limitiert, da die HPV-Testung nur zu einem Zeitpunkt erfolgte. Hierdurch lässt sich die zeitliche Sequenz zwischen Geschlechtsverkehr und HPV-Infektion nicht genau bestimmen. Für HPV-Impfprogramme ist jedoch die genauere Kenntnis des HPV-Infektionszeitpunkts, besonders auch die Rolle der nichtkoitalen Übertragungswege, von Bedeutung.

In einer amerikanischen Longitudinalstudie zu Risiken und protektiven Faktoren bei sexuell übertragbaren Infektionen wurden 387 Mädchen (14-17 Jahre) rekrutiert. Keines der Mädchen erhielt wegen Nichtverfügbarkeit während des Studienzeitraums eine HPV-Impfung. Die Befragung hinsichtlich der sexuellen Exposition erfolgte mittels persönlicher Interviews, Fragebögen und Tagebüchern ca. alle drei Monate. Bestand Kontakt zu einem Sexualpartner, wurden sie nach der Art der sexuellen Aktivität befragt. Ein regelmäßig entnommener Vaginalabstrich (vom Arzt oder selbst entnommen) wurde mittels PCR auf 37 verschiedene onkogene und nichtonkogene HPV-Genotypen untersucht. Von 387 Probandinnen gaben $36 \mathrm{zu}$ Studienbeginn keinen stattgehabten Koitus an; 14 der 36 wurden in der Folge aus verschiedenen Gründen aus der Analyse exkludiert. Die verbliebenen 22 Mädchen waren zu Studienbeginn 14,7 Jahre alt und überwiegend afroamerikanischer Abstammung. Die mittlere Nachverfolgungszeit betrug 5,2 $(4,5-8,2)$ Jahre. 21 der 22 Mädchen berichteten über einen ersten Koitus mit 16,5 Jahren. Die mittle- re Zeit von der Studienaufnahme bis zum ersten Koitus betrug 429 Tage. Bei 10 der 22 (45,2\%) Mädchen ließ sich bereits vor dem ersten Geschlechtsverkehr mindestens ein HPV-Genotyp im Vaginalabstrich nachweisen. Bei 8 der 10 HPVDNA-positiven Mädchen fand sich mindestens ein HPV-Hochrisikotyp. Bei 3 der 10 Mädchen ließen sich vakzine-präventable HPV-Typen $(6,11$, und 16) nachweisen. Insgesamt fanden sich bei den 10 Mädchen 29 HPV-Infektionen mit onkogenen (HPV-66, HPV-39, HPV-51, HPV16) sowie nichtonkogenen (HPV-42, HPV-62, HPV-6, HPV-11) HPV-Typen. Bei nichtkoitalen Sexualkontakten gaben sie Zungenküssse (81,8\%), Berührung der Genitalien des Partners oder Berührung 2012; online 26. Dezember, 10.1002/cncr.27914 der eigenen Genitalien durch den Partner $(54,6 \%)$ vor dem ersten Koitus an. Ferner wurde oro-genitaler Kontakt $(18,2 \%)$ sowie rezeptiver oro-genitaler Kontakt $(22,7 \%)$ genannt.

Fazit: Auf nichtkoitale Sexualkontakte wurde bei der Diskussion über das richtige Startalter der HPV-Impfung bisher nur wenig geachtet. Diese Longitudinalstudie belegt jedoch, dass nahezu die Hälfte der adoleszenten Mädchen schon vor dem ersten vaginalen Koitus durch diverse nichtkoitale Sexualpraktiken mit HPV infiziert sein können. Auch in früheren Untersuchungen wurde die Rolle von Fingern bei der Übertragung von HPV bestätigt. Die Studie verdeutlicht eindringlich die Bedeutung einer frühzeitigen HPV-Impfung. Beide HPVImpfstoffe sind ab dem 9. Lebensjahr zugelassen, werden aber wegen der geltenden STIKO-Empfehlung in der Regel erst ab dem 12. Lebensjahr angewendet.

Prof. Dr. Tino F. Schwarz

Shew ML et al.: Frequent detection of vaginal human papillomavirus prior to first sexual intercourse during longitudinal observation. J Infect Dis 2012 Dec 13; (Epub ahead of print)

\title{
Fünf Jahre nach Brustkrebsdiagnose normales Malignomrisiko
}

— Frauen, die einen Brustkrebs überlebt haben, erkranken vielfach erneut an einem primären Malignom. Ob die Malignominzidenz bei langzeitig überlebenden Patientinnen im Vergleich zur Altersgruppe erhöht ist, haben US-Forscher in einer Studie an 1.361 Patientinnen ( $\geq 70$ Jahre), die fünf Jahre nach der Diagnose eines Mammakarzinoms im Stadium I oder II noch lebten, zehn Jahre nachbeobachtet. 160 Brustkrebspatientinnen und 146 Frauen aus der Vergleichsgruppe erkrankten während der Nachbeobachtungszeit - im Mittel nach vier Jahren - an einem (weiteren) Primärtumor. Am häufigsten waren es Mammakarzinome ( $27 \%$ vs. $25 \%$ ), bei Brustkrebspatientinnen als kontralaterale Tumoren definiert. Die Brustkrebspatientinnen erkrankten seltener als die Kontrollen an Kolorektalkrebs $(11 \%$ vs. 20\%). Dafür trugen sie ein höheres Risiko für gynäkologische Malignome (7,5\% vs. 3,4\%). Signifikante Unterschiede im allgemeinen Krebsrisiko gab es nicht. Zwar lag die Rate in der Brustkrebsgruppe 17\% über jener der Vergleichsgruppe, der Unterscheid war aber nicht signifikant. Wären die ersten fünf Jahre nach der Brustkrebsdiagnose einbezogen worden, hätten sich die Resultate verändert. Denn währenddessen erhielten 6,8\% der Brustkrebsüberlebenden eine weitere Krebsdiagnose (v.a. Mammakarzinom oder andere gynäkologische Tumoren), aber nur 3,8\% der Frauen aus der Vergleichsgruppe.

Dr. Robert Bublak

Clough-Gorr KM et al. Incident Malignancies Among Older Long-Term Breast Cancer Survivors and an Age-Matched and Site-Matched Nonbreast Cancer Comparison Group Over 10 Years of Follow-up. Cancer 